\title{
CANINE IDIOPATHIC CHRONIC HEPATITIS
}

\section{Corresponding author:}

Nick Bexfield BVetMed PhD DSAM DipECVIM-CA FRSB AFHEA MRCVS

Clinical Associate Professor in Small Animal Medicine and Oncology

School of Veterinary Medicine and Science

University of Nottingham

Leicestershire

UK

nick.bexfield@nottingham.ac.uk

The author has nothing to disclose.

\section{Key Words}

Idiopathic; hepatitis; canine; diagnosis; management;

prognosis

\section{Synopsis/Abstract}

\section{Key Points}

- Canine idiopathic hepatitis is a common disease, categorized histologically by presence of hepatocellular apoptosis or necrosis, a variable mononuclear or mixed inflammatory cell infiltrate, regeneration and fibrosis. 
- Clinical signs are vague and non-specific, but there are known breed, age and gender predispositions.

- Results of clinical pathology are non-specific, but usually include elevations in liver enzymes and function impairment; a liver biopsy is required for diagnosis.

- Management involves around the use of an anti-inflammatory dose of glucocorticoids and other supportive and symptomatic therapies including ursodeoxycholic acid, antioxidants, diuretics, and diet.

- The prognosis is variable, but there are known prognostic indicators, especially the presence of portal hypertension. 


\section{Introduction}

Historically, the term canine chronic hepatitis $(\mathrm{CH})$ has been poorly defined and used to describe a variety of inflammatory liver diseases. However, the World Small Animal Veterinary Association's (WSAVA) Liver Standardization Group have relatively recently produced standardized criteria for the histological diagnosis of canine liver diseases, including $\mathrm{CH}$. They define $\mathrm{CH}$ by the presence of hepatocellular apoptosis or necrosis (figure 1), a variable mononuclear or mixed inflammatory cell infiltrate (Figure 2), regeneration (figure 3) and fibrosis (figure 4). ${ }^{1}$ However, it is clear there are variations in histological appearance between breeds..$^{2,3}$

Hepatic copper accumulation is an important cause of canine $\mathrm{CH}$ (please see Hille Fieten and Karen Dirksen's "Canine copper-associated hepatitis," in this issue). However, for many cases where copper accumulation has been ruled out, the cause of $\mathrm{CH}$ remains unknown despite the patient undergoing a complete diagnostic workup. These dogs are said to have idiopathic chronic hepatitis. This article reviews the current theories regarding the etiopathogenesis of canine $\mathrm{CH}$ other than copper accumulation, as well as the clinical features, diagnostic findings, and management of idiopathic $\mathrm{CH}$.

\section{Pathophysiology}

The development of fibrosis is the key pathological change which leads to the development of chronic liver disease, including canine idiopathic $\mathrm{CH}^{8}$ The hepatic stellate cell, also known as the Ito cell or lipocyte, is central in the development of fibrosis in humans and rats, ${ }^{8-11}$ and evidence also suggests its involvement in the pathogenesis of hepatic fibrosis in

dogs. ${ }^{6}$ Canine hepatic stellate cells reside in the space of Disse, between the sinusoidal endothelial cells and the hepatocytes. ${ }^{12}$ In normal human and rat liver they are the major storage site of vitamin $\mathrm{A}$, and also synthesize extracellular matrix components, matrix 
metalloproteinases, cytokines, and growth factors. ${ }^{8,9}$ Although their function in canine liver has not been extensively studied, they likely possess similar roles. In human chronic liver injury they are stimulated to transform into collagen-secreting, activated stellate cells which express smooth muscle-specific $\alpha$-actin, and secrete high-density matrix and collagen into the space of Disse. ${ }^{11}$ In most cases of human and experimental animal chronic liver disease, the simulation of stellate cells is believed to be indirect via the release of cytokines from inflammatory cells, although some substances act on them directly to stimulate fibrosis. ${ }^{8}$ For instance, activated Kupffer cells in the human liver secrete transforming growth factor $\beta 1$ (TGF- $\beta$ ), the most potent fibrogenic factor, and also other cytokines such as platelet-derived growth factor. ${ }^{8,11}$ Inflammatory mediators released from neutrophils, lymphocytes, platelets, and necrotic hepatocytes have also been implicated in the pathogenesis of hepatic fibrosis in humans,${ }^{11}$ but their contribution to the development of fibrosis in canine liver disease has not been investigated. Damage to cells and mitochondrial membranes by bile acids in cholestatic liver disease also plays a role in producing oxidant damage and stimulating cytokine release. ${ }^{13}$ The formation of fibrous tissue within the liver leads to the development of intrahepatic portal hypertension by altering sinusoidal tone and blood flow. ${ }^{9}$ The development of portal hypertension results in many of the complications of chronic liver disease such as ascites and gastrointestinal ulceration.

\section{Etiology}

Although there are several known causes of canine $\mathrm{CH}$, the majority of dogs have idiopathic disease. ${ }^{3,14,15}$ A limited number of infectious causes are documented, and include canine adenovirus type $\mathrm{I}(\mathrm{CAV}-1)^{16}$ and Leptospira spp. ${ }^{17,18}$ Although initially postulated as a canine hepatitis causing virus, the recently identified canine homology of human hepatitis $\mathrm{C}$ virus, canine hepacivirus (CHV), ${ }^{19}$ does not appear to be involved in the etiology of canine $\mathrm{CH}$ in the United Kingdom ${ }^{20}$ or the Netherlands. ${ }^{21}$ Although bacteria, especially those originating 
from the gastrointestinal tract, are known to cause canine cholangitis/cholangiohepatitis, ${ }^{22,23}$ their role in the etiology of $\mathrm{CH}$ is unknown. Toxins and drug reactions usually cause acute, necrotizing hepatitis ${ }^{24}$ but some such as phenobarbitone can cause $\mathrm{CH}^{25}$ Defects in copper metabolism leading to the development of $\mathrm{CH}$ have been described in several breeds of dog. These include true 'copper storage' disease, where the primary initiating event is a build up of copper leading to secondary hepatic damage, and 'copper-associated' disease, in which copper accumulates secondary to an underlying liver disease including $\mathrm{CH}$. Copper storage disease was first described in the Bedlington terrier over three decades ago, ${ }^{26}$ and since then has been reported in the Dalmatian, ${ }^{27}$ Doberman pinscher, ${ }^{28}$ and Labrador retriever. ${ }^{29}$ Further details on the role of copper in the etiology of $\mathrm{CH}$ can be found in Hille Fieten and Karen Dirksen's "Canine copper-associated hepatitis," and Penny J Watson's "Canine Breed Specific Hepatopathies," in this issue. To date, no data exists to convincingly demonstrate the presence of an underlying autoimmune etiology to canine $\mathrm{CH}$. Low concentrations of antinuclear antibodies were present in some dogs with $\mathrm{CH}$, and the occasional dog had high circulating concentrations of antibodies to liver membrane proteins. ${ }^{30}$ In another study, 10 of 21 dogs with $\mathrm{CH}$ had antibodies to anti-liver membrane protein. ${ }^{31}$ More recently, autoantibodies against liver proteins were found in sera of Doberman pinschers with $\mathrm{CH}$ before the onset of clinical signs. ${ }^{32}$ Using an indirect immunofluorescence assay on cryostat sections of mouse liver small numbers of Doberman's with $\mathrm{CH}$ had evidence of antinuclear antibodies, ${ }^{33}$ and $\mathrm{IgG}$ autoantibodies against histones. ${ }^{34}$ However, the question that remains from all these studies is whether these autoantibodies are the primary cause of the disease, or a secondary phenomenon. Finally, the association of certain susceptibility and resistance major histocompatibility complex (MHC) class II haplotypes in the Doberman pinschers, ${ }^{35}$ and English springer spaniels (ESS) ${ }^{36}$ clearly indicates a role of the immune system in disease development, but does not necessarily point to an autoimmune etiology. 


\section{Signalment}

Canine $\mathrm{CH}$ is common in the United Kingdom with a reported postmortem prevalence of $12 \%$ in a first opinion practice setting. ${ }^{37}$ There are several reports of apparent breed, age and gender predispositions, although these vary depending on geographic location and also the time the study was performed. Moreover, not all dogs in these studies necessarily had idiopathic $\mathrm{CH}$, as comprehensive work to eliminate known causes was not always performed. An early study performed approximately two decades ago on a Swedish population of dogs, and prior to the establishment of the WSAVA criteria for the histological diagnosis of $\mathrm{CH}$, demonstrated that the American and English cocker spaniel, West Highland white terrier, Labrador retriever, Doberman pinscher and Scottish terrier had an increased risk for $\mathrm{CH} .{ }^{38} \mathrm{In}$ this study, there was a male predisposition in English and American cocker spaniels which were a mean of 5 years old, and female predisposition in Labrador retrievers which were a mean of 6.9 years old at presentation. A more recent study performed in the UK assessed for breed predilections to $\mathrm{CH}$ by comparing the signalment of 551 cases to that of a large control population. ${ }^{15}$ From a total of 61 breeds, the American cocker spaniel, Cairn terrier, Dalmatian, Doberman pinscher, English cocker spaniel, English springer spaniel, Great Dane, Labrador retriever, and Samoyed were found to be at increased risk for developing $\mathrm{CH}$ (see Table 1). In this study, the median age for all breeds with $\mathrm{CH}$ was 8 years (range, 7 months to 16 years), and the female to male ratio was 1.5:1.. In Japan, a predilection of female Labrador retrievers and Doberman pinschers for $\mathrm{CH}$ was reported, and the median age of dogs was 8 years and 7 months. ${ }^{39}$ The mean age of 43 dogs with idiopathic $\mathrm{CH}$ in one retrospective study was 7.7 years (range 0.4 to 14.2 years), although there was no data on apparent breed or gender predispositions. ${ }^{3}$ For further details of breed, age and gender predispositions to canine idiopathic CH please see Penny J Watson's “Canine Breed Specific Hepatopathies,” in this 
issue.

\section{History and physical examination findings}

The liver has tremendous functional and structural reserve, and a significant loss of normal hepatic mass can occur with minimal or no clinical signs. ${ }^{40}$ The liver also has a remarkable capacity to regenerate following hepatic injury, providing the damage is not too severe or ongoing, ${ }^{41}$ and overt liver failure does not develop until at least $70-80 \%$ of functional capacity is lost. ${ }^{42}$ Historical signs in dogs with idiopathic $\mathrm{CH}$ vary and are usually nonspecific and insidious in onset. The more frequently reported include inappetence, lethargy, weight loss, vomiting, and diarrhea. ${ }^{42,43}$ Some dogs with idiopathic $\mathrm{CH}$ can be asymptomatic, or alternatively may present with signs of 'acute' disease. ${ }^{2}$ The duration of clinical signs also varies, and in 43 dogs with idiopathic $\mathrm{CH}$, were present for a median of 4.3 weeks prior to presentation (range, 0.5-14.0 weeks). ${ }^{3}$ In a study of 101 dogs with both acute hepatitis and $\mathrm{CH}$, the most commonly identified signs were lethargy $(\mathrm{n}=56)$, anorexia $(\mathrm{n}=56)$, vomiting $(n=48)$, polyuria and polydipsia $(n=27)$ weight loss $(n=28)$ and diarrhea $(n=23){ }^{3}$ In 68 ESS with idiopathic $\mathrm{CH}$, clinical signs at presentation included lethargy $(\mathrm{n}=63)$, decreased appetite $(n=58)$, vomiting $(n=34)$, weight loss $(n=31)$, diarrhea $(n=21)$, and polydipsia $(n=21){ }^{2}$ In 16 Labrador retrievers with $\mathrm{CH}$, signs included vomiting $(\mathrm{n}=9)$, lethargy $(\mathrm{n}=8)$, weight loss $(n=6)$, decreased appetite $(n=5)$, diarrhea $(n=2)$, and polyuria/polydipsia $(n=2) .{ }^{44}$

Physical examination findings are also very variable and likely depend on the duration and severity of disease. Physical examination findings in 101 dogs with both acute and $\mathrm{CH}$ included jaundice $(n=24)$, abdominal distention $(n=21)$, signs of hepatic encephalopathy (HE) $(n=22)$, hepatomegaly $(n=17)$, and abdominal pain $(n=8) \cdot{ }^{3}$ Physical examination in 68 ESS with idiopathic $\mathrm{CH}$ revealed icterus $(n=37)$, hyperthermia $(n=24)$, poor body condition $(n=23)$, ascites $(n=17)$, and abdominal pain $(n=9) .{ }^{2}$ A range of concurrent diseases, including 
those of the endocrine, renal, cardiovascular, musculoskeletal, dermatological and neurological systems, are also sometimes documented in dogs with idiopathic $\mathrm{CH}^{2,3}$

\section{Clinical pathology}

\section{Serum biochemistry}

Clinical pathology is the next step in investigating the dog with suspected idiopathic $\mathrm{CH}$. Findings usually include elevations of liver enzyme activities, especially hepatocellular enzymes released as a result of liver cell damage, namely alanine aminotransferase (ALT) and aspartate aminotransferase (AST). In addition, the markers of cholestasis, alkaline phosphatase (ALP) and gamma-glutamyl transferase (GGT) are invariably elevated. There is a wide variation in absolute values of the various clinical pathology tests, and very occasionally a dog with advanced idiopathic $\mathrm{CH}$ may have liver enzyme activity within the reference interval. In addition to elevations in liver enzyme activities, due to the development of fibrosis, liver function in dogs with idiopathic $\mathrm{CH}$ is progressively compromised. Markers of reduced liver function include decreased serum albumin, urea, glucose, and coagulation factors, while serum bile acids, ammonia and bilirubin may increase. Of course, none of these tests are specific for canine idiopathic $\mathrm{CH}$, so should always be interpreted in the light of other clinical and clinicopathological findings.

In a variety of breeds of dog with idiopathic $\mathrm{CH}$ serum, serum ALP activity was increased in 32/33 (mean: $660 \mathrm{U} / \mathrm{L}$ ) and ALT in 16/17 (mean: $403 \mathrm{U} / \mathrm{L}$ ). ${ }^{3}$ Albumin was decreased in 14/24 (mean: $26.0 \mathrm{~g} / \mathrm{L}$ ), and glucose in 2/8 (mean: $4.9 \mathrm{mmol} / \mathrm{L}$ ). In 32/35 dogs, bile acids were increased (mean: $75.7 \mu \mathrm{mol} / \mathrm{L}$ ), while resting ammonia was increased in 1/7 dogs (mean: $33.9 \mu \mathrm{mol} / \mathrm{l})$. In $68 \mathrm{ESS}$ with idiopathic $\mathrm{CH}$, serum ALT and ALP activities were elevated in all dogs with median values of 690 and $821 \mathrm{U} / \mathrm{L}$, respectively. ${ }^{2}$ Serum AST and 
GGT activities were elevated in $75 \%$ and $63 \%$ of cases with median values of 361 and 26 U/L. In $76 \%$ of cases, resting bile acids were increased with a median of $87 \mu \mathrm{mol} / \mathrm{L}$, and bilirubin was increased in $69 \%$ of cases with a median of $77 \mu \mathrm{mol} / \mathrm{L}$. Albumin and urea, were reduced in 38 and $22 \%$ of cases, respectively. In 24 Labrador retrievers with $\mathrm{CH}$, all dogs had increases in serum activity of one or more hepatobiliary enzyme, with increased ALP ( $n=21)$ $\operatorname{ALT}(n=20), \operatorname{AST}(n=20)$ and GGT $(n=9) .{ }^{44}$ Hyperbilirubinemia and hypoalbuminemia were present in $45 \%$ and $21 \%$ of dogs respectively, pre- or postprandial serum bile acid concentrations were increased in 2 of 4 cases, and one of six dogs tested had hyperammonemia. $^{44}$

\section{Hematology}

Non-specific qualitative and quantitative abnormalities in erythrocytes may be present in dogs with idiopathic $\mathrm{CH}$, in combination with alterations in white cell and platelet numbers. Alterations to red cell morphology may also include the presence of acanthocytes and codocytes. Dogs with idiopathic $\mathrm{CH}$ may have a neutrophilic leukocytosis, due to stress or an inflammatory response, and thrombocytopenia may develop due to platelet sequestration or increased destruction.

In 21 dogs with idiopathic $\mathrm{CH}$, the hematocrit was decreased in 11 , with a mean of $0.40 \mathrm{~L} / \mathrm{L}$ and thrombocytopenia was identified in $4 / 10$, with a mean platelet count of $212 \times 10^{9} / \mathrm{L} .^{3}$ Eleven of 24 dogs had evidence of a leukocytosis (mean: $14.8 \times 10^{9} / \mathrm{L}$ ) with segmented neutrophils present in 10/24 dogs. The median white blood cell count was $16.1 \times 10^{9} / \mathrm{L}$ in 68 ESS with idiopathic $\mathrm{CH}$, and $77 \%$ of cases had an elevated white blood cell count. ${ }^{2}$ Results of hematology from 24 Labrador retrievers with $\mathrm{CH}$ included an increased WBC (n=9), lymphopenia $(n=7)$, neutropenia $(n=2)$, left shift $(n=2)$, and monocytosis $(n=2) .{ }^{44}$ The median 
hematocrit was 46\%, and seven dogs were anemic (median, 31\%; range, 23-35\%). Four dogs had thrombocytopenia (median: $128 \times 10^{3} / \mathrm{mL}$; range, $67-177 \times 10^{3} / \mathrm{mL}$ ), and one dog had a thrombocytosis.

\section{Urinalysis}

Non-specific abnormalities may also be present in the urine of dogs with idiopathic $\mathrm{CH}$, including reduced specific gravity as a result of PU/PD, bilirubinuria, and increased urobilinogen. Urate crystals are occasionally seen. A paucity of data exists on results of urinalysis in dogs with idiopathic $\mathrm{CH}$, but in 14 Labrador retrievers with $\mathrm{CH}$ of a variety of causes, bilirubinuria was identified in seven dogs, transient glucosuria without hyperglycemia in three, and proteinuria in two. ${ }^{44}$

\section{Hemostasis}

The canine liver plays an important role in maintaining hemostasis, and the resulting decreased function in idiopathic $\mathrm{CH}$ may result in abnormalities of coagulation (please see Cynthia RL Webster's “Hemostatic Disorders Associated with Hepatobiliary Disease," in this issue). Hepatocytes not only produce fibrinogen, prothrombin and the majority of the coagulation factors, but are also responsible for the activation of the vitamin K-dependent factors and protein $\mathrm{C} .{ }^{45}$ It is therefore essential to assess coagulation prior to a liver biopsy. In humans with chronic hepatocellular disease, levels of vitamin K-dependent clotting factors, particularly factor VII and protein C, may decrease. ${ }^{46}$ However, increased activation may also occur in patients with liver diseases and a consumptive coagulopathy, leading to disseminated intravascular coagulation (DIC), has been reported in patients with end stage liver disease. ${ }^{46}$

In one study of dogs with $\mathrm{CH}$ of various causes, although both mean prothrombin time (PT) 
and activated partial thromboplastin time (aPTT) were above upper reference values, only PTT was significantly prolonged in dogs with $\mathrm{CH}$ and cirrhosis. ${ }^{47}$ The mean activities of factors II, V, VII, VIII, IX, X, XI, and XI activities, and protein C were lower in dogs with $\mathrm{CH}$ and cirrhosis compared to dogs with $\mathrm{CH}$ alone. ${ }^{47}$ Mean D-dimer concentrations were not significantly increased in dogs with $\mathrm{CH}$ or $\mathrm{CH}$ and cirrhosis. ${ }^{47}$ In 34 dogs with idiopathic $\mathrm{CH}$, an elevated PT (mean: 10.1 seconds) was identified in 14 dogs, an elevated PTT (mean: 17.1 seconds) in 12 , and reduced fibrinogen concentration in eight (mean: $2.5 \mathrm{~g} / \mathrm{L}){ }^{3}$ Prothrombin time and aPTT were measured in 49 ESS with idiopathic $\mathrm{CH}$ and were increased in $24 \%$ and $21 \%$, respectively. ${ }^{2}$ Seven of 21 Labrador retrievers with $\mathrm{CH}$ had prolonged PTT and nine dogs had prolonged PT. ${ }^{44}$

\section{Diagnostic imaging}

Diagnostic imaging, including abdominal radiography and ultrasonography, is an important part of the investigation of a dog with suspected idiopathic $\mathrm{CH}$, and is typically performed in combination with clinical pathology. Abdominal radiography can be used to assess liver size, position and shape, and to check for the presence of other abdominal pathology. ${ }^{48}$ As a general rule, liver size is reduced in dogs with chronic diseases such as idiopathic $\mathrm{CH}$. Splenomegaly may also be present in dogs with $\mathrm{CH}$ and portal hypertension. Abdominal radiography in dogs with idiopathic $\mathrm{CH}$ and ascites secondary to portal hypertension or hypoalbuminemia is generally unhelpful because the fluid obscures abdominal detail. However, radiographic evaluation is poorly sensitive and specific for the diagnosis of idiopathic $\mathrm{CH}$; the liver may appear radiographically normal even when severely diseased. Few studies report the radiographic findings in dogs with idiopathic $\mathrm{CH}$, although in $33 \mathrm{ESS}$, 27 had reduced liver size and 16 had splenomegaly. ${ }^{2}$ 
Ultrasonography is an established method for examining the canine liver, and it enable an assessment of its size, shape, echogenicity and echotexture, as a means of detecting lesions affecting the hepatic parenchyma. However, ultrasonography is generally considered unsuitable as a sole method for diagnosis because of the large overlap in the ultrasonographic appearance between different hepatic diseases, including $\mathrm{CH}^{49,50}$ In a recent study, a marked variability in ultrasonographic appearance of lesions was observed for all diagnoses, including hepatitis, and no statistically significant associations between ultrasonographic appearance and diagnosis were found. ${ }^{49}$ The sensitivity for the diagnosis of hepatitis of all causes in this latter study was $48 \%$, with $10 \%$ of dogs having multifocal hypoechoic parenchymal lesions, and $10 \%$ showing diffuse parenchymal heterogenicity. Cirrhosis, the end stage of idiopathic chronic hepatitis, may be associated with a small, irregularly marginated liver; extensive hepatic fibrosis usually results in diffusely increased hepatic echogenicity, often with hypoechoic regeneration nodules scattered throughout the parenchyma and bulging from the hepatic margins. ${ }^{51}$ Portal hypertension is a common sequel to hepatic fibrosis, resulting in ascites and the development of acquired portosystemic shunts, which are occasionally visible on ultrasonographic examination.

In 67 dogs with idiopathic and copper-associated $\mathrm{CH}, 15$ had no identifiable ultrasonographic abnormalities, hepatic size was assessed as normal $(n=26)$, small $(n=26)$ and enlarged $(n=8){ }^{3}$ Hepatic structure was found to be normal in 27 , irregular in 31 , and 11 had increased echogenicity. Nodular processes were observed in $16 \mathrm{dogs}$, and ascites was present in 18 . On ultrasonographic examination of $68 \mathrm{ESS}$ with $\mathrm{CH}$, the liver appeared small in 49 dogs and normal in size in the remainder. ${ }^{2}$ Changes in hepatic parenchymal echogenicity were present in $62 \operatorname{dogs}$ and included hypoechogenicity $(n=12)$, hyperechogenicity $(n=10)$, or a combination of hypo- and hyperechogenicity $(n=40)$ when compared with the echogenicity of 
the spleen. Six dogs had a normal appearance to the liver on ultrasound.

\section{Histopathology}

It is not possible to make a definitive diagnose of idiopathic $\mathrm{CH}$ from results of signalment, history, physical examination, clinical pathology or diagnostic imaging. In some cases, a tentative diagnosis can be made, but the gold standard for diagnosis is the histopathological evaluation of liver tissue. This enables subjective descriptors of severity of inflammation and a subjective assessment about the amount of fibrosis; these may be used prognostically and therapeutically. Histopathology is important to rule out significant copper accumulation as a cause of $\mathrm{CH}$. Liver histology will also give vital information to help establish the most appropriate therapy, and without a biopsy, therapy of idiopathic $\mathrm{CH}$ will be at best nonspecific and at worst counter-productive.

Many methods are available for collection of liver tissue, and the method used will depend on clinician preference, availability of equipment, technical skill, cost and the clinical stability of the patient (please see Jonathan A. Lidbury's "Getting the Most out of Liver Biopsy," in this issue). Cytological evaluation of liver aspirates is of limited diagnostic accuracy in the diagnosis of idiopathic $\mathrm{CH}$, and is therefore not recommended. ${ }^{52,53}$ This relates to both the importance of liver architecture in the categorization of the diagnosis, and the chances of missing inflammatory cells where there are areas of fibrosis or if the distribution is nonuniform. In one study inflammatory disease was accurately identified cytologically in only 5 of 20 dogs. ${ }^{53}$ The only real absolute contraindication for liver biopsy is severe coagulopathy (please see Jonathan A. Lidbury's "Getting the Most out of Liver Biopsy," in this issue).

Histopathological evaluation should be carried out in accordance with criteria produced by 
the WSAVA Liver Standardization Group. ${ }^{1}$ The standard histochemical stain used in the assessment of liver tissue is hematoxylin and eosin, but consideration should be given to the use of additional stains for specific features such as Masson's trichrome (connective tissue), Perls' Prussian blue (ferritin), Fouchet's (bile pigments), and periodic acid-Schiff (polysaccharides). In addition, to rule out primary copper accumulation as a cause of $\mathrm{CH}$, specific histochemical stains for copper, such as rubeanic acid or rhodanine, should be used, especially in at risk breeds. An objective grading and scoring system for $\mathrm{CH}$, akin to those used in human medicine, ${ }^{4,5}$ would enable better characterization of the canine disease to improve studies into etiology, response to treatment and prognosis. Although bacteria do not appear to be a significant cause of $\mathrm{CH}$, before a diagnosis of the idiopathic disease is made, culture of bile or liver tissues could be performed. Although not routinely undertaken, fluorescent in situ hybridization (FISH) could also be considered for the identification of intra-hepatic bacteria causing $\mathrm{CH} .{ }^{55}$

Findings on histopathological examination will depend on the duration and severity of disease. The inflammatory cell infiltrate in dogs with $\mathrm{CH}$ has been poorly characterized, primarily due to a lack of canine-specific reagents. In one study, high numbers of CD3+ lymphocytes were found in liver tissue of $\operatorname{dogs}$ with $\mathrm{CH},{ }^{6}$ although the etiology of these cases was not clear. These cells were closely apposed to degenerated hepatocytes and a positive correlation between necrosis and the number of portal and lobular CD3+ lymphocytes was noted. The hepatic T lymphocyte phenotype has also been characterized in a dog with $\mathrm{CH}$ before and after treatment. ${ }^{7}$ Before treatment, numerous CD3+ lymphocytes were present in the liver, and the ratio of $\mathrm{CD} 4+/ \mathrm{CD} 8+$ was high, and after treatment, CD3+ lymphocyte infiltration was reduced along with the ratio of CD4+/CD8+ cells. In ESS with idiopathic $\mathrm{CH}$, the predominant inflammatory cell type was the lymphocyte, with all dogs having lesser 
number of plasma cells. ${ }^{2}$ Forty-five of 68 dogs also had a neutrophilic inflammatory cell infiltrate, but there were always less neutrophils than lymphocytes. The inflammatory cell infiltrate was present both in the portal areas and throughout the hepatic parenchyma. In this latter study, all dogs had evidence of increased fibrous connective tissue on reticulin staining and 38 dogs had bridging fibrosis and of these 22 had cirrhosis. Cases with bridging fibrosis had combinations of portal-portal $(n=30)$, portal-central $(n=24)$, and central-central $(n=18)$ fibrosis. In 24 Labrador retrievers with $\mathrm{CH}$ of various causes, all dogs had mild-to-moderate infiltrates of lymphocytes or macrophages, which were most prominent in the portal areas. ${ }^{44}$ Thirteen dogs also had a neutrophilic component, and twenty-one had degenerative hepatocellular changes. Twenty-one dogs had evidence of increased fibrous tissue on hematoxylin and eosin staining, and on examination of Masson's trichome stained sections, six dogs had bridging fibrosis, and four had cirrhosis. In 43 dogs with idiopathic $\mathrm{CH}$, cirrhosis was observed in 23 , including macronodular cirrhosis in seven. ${ }^{3}$

\section{Management}

As the etiology of idiopathic $\mathrm{CH}$ is currently not understood, specific therapies are not possible. Non-specific therapies seem to, however, make a significant difference to the quality of life and probably also the survival time of affected dogs. Hepatocytes have a remarkable capacity for regeneration, which means that early diagnosis and therapy has the potential to reverse disease mechanisms. A key aim of therapy in canine idiopathic $\mathrm{CH}$ is to inhibit fibrosis, which, if left to progress, will ultimately lead to functional impairment. Therapy aimed at addressing clinical signs of liver disease, including ascites, gastrointestinal ulceration, and HE is also an important part of therapy of the dog with idiopathic $\mathrm{CH}$. In addition, careful dietary management is very important. Unfortunately there is a paucity of controlled studies on clinical efficacy and pharmacokinetics of the commonly used drugs in 
canine idiopathic $\mathrm{CH}$. As a result, many of our current management strategies are either derived from human hepatology, from veterinary clinical experience, or originate from lowquality veterinary clinical studies and anecdotal reports.

\section{Glucocorticoids}

Glucocorticoids have anti-inflammatory, immune-modulating, and antifibrotic properties ${ }^{56}$. They have a potent indirect antifibrotic action via reducing prostaglandin and leucotriene production from inflammatory cells, and a weak direct anti-fibrotic action by inhibiting mRNA and enzymes. However, glucocorticoids are not without adverse effects, and these can be very severe and potentially life-threatening in some dogs with idiopathic $\mathrm{CH}$. Adverse effects include increased protein catabolism, fluid retention, gastrointestinal ulceration, an increased risk of infection. Glucocorticoids should be used cautiously in dogs with bridging fibrosis or cirrhosis, as these changes can be associated with portal hypertension, ascites, and/or gastrointestinal ulceration.

There have been limited studies evaluating the use of glucocorticoids in dogs with idiopathic $\mathrm{CH}$. In an early study, prednisolone was used in $151 \mathrm{dogs}$ with $\mathrm{CH}$ at a dose of $2.2 \mathrm{mg} / \mathrm{kg} / \mathrm{day}$ $P O$ for 7-14 days, and resulted in a significantly increased survival time when compared to untreated dogs. ${ }^{57}$ Moreover, complete remission occurred in some dogs with no relapse. However, this study was retrospective and uncontrolled, and it is unknown how many of the dogs would fit the criteria for idiopathic $\mathrm{CH}$ according to current WSAVA criteria. In a more recent study, 36 dogs with idiopathic $\mathrm{CH}$ were treated with prednisolone at a dose of 1 $\mathrm{mg} / \mathrm{kg} / \mathrm{day} P O$ for at least six weeks, and at follow up 11 dogs were in complete remission, eight dogs had recurrent clinical signs, and 17 dogs had residual disease. The use of glucocorticoids has also been reported in retrospective studies of dogs with idiopathic $\mathrm{CH}$, although as it is likely that varying doses were used, and most dogs received other 
medications, it is difficult to draw conclusions on their efficacy. ${ }^{2,44}$ It appears that clinicians are being selective in the cases they treat with glucocorticoids; for instance in a retrospective study of 43 dogs with idiopathic $\mathrm{CH}$, prednisone was not used in 12 dogs due to the presence of only mild inflammation on histology. ${ }^{3}$

The results of these studies therefore do support the use of glucocorticoids in dogs with $\mathrm{CH}$. In the author's opinion, glucocorticoids are indicated in the therapy of idiopathic $\mathrm{CH}$ when there is biopsy evidence of ongoing inflammation and this is associated with mild to moderate fibrosis. The glucocorticoid most commonly used is prednisolone rather than prednisone, as the latter drug needs to be metabolized into prednisolone by the liver. The ideal dose remains unknown, but as there is currently no evidence for an immune-mediated etiology for canine $\mathrm{CH}$, an immunosuppressive dose does not appear to be warranted. Moreover, this dose is likely to be associated with more severe adverse effects. The author currently uses an anti-inflammatory dose of $1 \mathrm{mg} / \mathrm{kg} /$ day $P O$. The duration, or method of dose reduction in dogs with idiopathic $\mathrm{CH}$ is also not known. In human patients, glucocorticoids are continued for at least six months beyond remission, and in some cases life-long treatment is given. It is often difficult to assess remission in dogs, particularly as glucocorticoids induce hepatic enzymes and so confuse attempts to follow the disease clinicopathologically. Repeat liver biopsy can be very useful, although they are infrequently performed in our canine patients. The length of therapy therefore remains empirical and some animals remain on life-long therapy. In this situation the aim is to use a low alternate day dose. There is little data to recommend the use of alternative immune modulating drugs, such as azathioprine and cyclosporine, in canine idiopathic $\mathrm{CH}$.

\section{Antifibrotics}


As detailed above, progressive fibrosis in idiopathic $\mathrm{CH}$ ultimately leads to reduced function and many of the clinical consequences of advanced liver disease. As yet, there are no widely available therapies to inhibit the action of inflammatory cytokines, such as TGF- $\beta$, on hepatic stellate cells; the main stimulus for fibrous tissue formation. The primary way to inhibit fibrosis is to treat the underlying disease mechanism, hence why anti-inflammatory drugs are usually indicated in the management of canine idiopathic $\mathrm{CH}$. In addition to the anti-fibrotic action of glucocorticoids, other drugs have been reported to have weak direct or indirect antifibrotic actions including zinc and vitamin $\mathrm{E}$.

Colchicine is a more specific antifibrotic; it is an alkaloid that binds to $\beta$-tubulin, thus inhibiting self-assembly and polymerization of microtubules and interfering with several cellular functions. ${ }^{58}$ In addition, colchicine modulates the production of chemokines and prostanoids, inhibits neutrophil and endothelial cell adhesion molecules and eventually it decreases neutrophil degranulation, chemotaxis and phagocytosis, thus reducing the initiation and amplification of inflammation. ${ }^{59}$ Although historically it has been used as a hepatic antifibrotic in human medicine, in more recent large clinical studies it appears not to be effective, and so the hunt still goes on for efficacious antifibrotics. ${ }^{60}$ There is limited evidence of its use in dogs,${ }^{61,62}$ and only anecdotal reports of its use in the management of idiopathic $\mathrm{CH}$. Adverse effects, including bone marrow suppression, neurological signs, anorexia, and diarrhea, are seen relatively commonly. As such, the author does not use colchicine in the management of idiopathic $\mathrm{CH}$.

\section{Ursodeoxycholic acid}

Ursodeoxycholic acid (UDCA) is a natural hydrophilic bile acid present in the biliary system, but is also synthesized and manufactured commercially. Ursodeoxycholic acid is nontoxic to 
the liver, whereas the less hydrophilic bile acids such as lithocolic acid can be highly toxic. Bile acids exert their toxicity by inducing apoptosis of hepatocytes, and disrupting the mitochondrial electron-transport chain leading to the formation of free radicals and oxidative damage to cells. Ursodeoxycholic acid exerts its beneficial effects in the liver by preventing cells from entering apoptosis and preventing mitochondrial damage, likely by displacing toxic hydrophobic bile acids. ${ }^{63-65}$ Ursodeoxycholic acid is also a cholerectic i.e. it stimulates bile flow. In addition it has also been shown to have immune-modulatory actions by reducing immunoglobulin and interleukin production and expression of major histocompatibility complex-1 on hepatocytes. Studies show an additional anti-oxidant activity of UDCA due to increased production of glutathione (GSH).

Although UDCA appears to be used very widely in veterinary practice, little data exists on its efficacy in dogs. There is a single case report of a dog with severe cholestasis secondary to suspected idiopathic $\mathrm{CH}$ whose sole treatment was UDCA. ${ }^{66}$ In this dog, serum activities of liver enzymes and concentrations of cholesterol and total bilirubin all decreased over the course of seven months of treatment. Concomitant with this improvement, the composition of the serum bile acids changed from the hydrophobic, toxic, endogenous bile acids to the less toxic hydrophilic bile acids. Although there are also several retrospective studies of dogs with idiopathic $\mathrm{CH}$ in which UDCA was used, dogs typically received multiple therapies, making it difficult to determine the specific effect of UDCA. At a dose of $15 \mathrm{mg} / \mathrm{kg} / \mathrm{day} P O$, UDCA appears to be safe, with no adverse effects reported in the literature. As most dogs with idiopathic $\mathrm{CH}$ have bile stasis, and thus would likely benefit from UDCA, the author uses it in all cases.

\section{Antibiotics}


As bacteria are not involved in the etiology of dogs with idiopathic $\mathrm{CH}$, antibiotics are generally not warranted. However, due to compromised reticuloendothelial cell function in chronic liver disease,${ }^{67}$ bacterial infections may be a secondary complication of idiopathic $\mathrm{CH}$. The mainstay of antibiotic use in dogs with idiopathic $\mathrm{CH}$ is in the management of $\mathrm{HE}$, which arises with the development of portal hypertension and acquired shunts (please see Adam G. Gow's “Hepatic Encephalopathy,” in this issue). In the author's experience, clinical HE is relatively uncommon, even in dogs with advanced liver disease. If there are signs of HE, oral antibiotics to suppress bacterial populations that produce gut derived encephalopathic toxins are warranted, and these are usually used with lactulose for a synergistic effect. ${ }^{68}$ Suitable antibiotics effective against anaerobic organisms include amoxicillin, metronidazole, and neomycin. Metronidazole and amoxicillin have an added advantage over neomycin as they are systemically absorbed so may protect against bacteremia.

\section{Antioxidants}

Oxidative stress and damage to cells by reactive oxygen species is an important disease mechanism. Free radicals take up electrons from neighboring molecules, which results in oxidative damage to proteins, lipids, and DNA. In liver disease, oxidant stress is increased due to the effects of inflammation, reduced blood flow, and mitochondrial damage by refluxed bile acids. Normal cellular protective mechanisms against oxidative damage include GSH. Most of the evidence for antioxidants is in acute liver injury, especially that due to hepatotoxins. ${ }^{69}$ Oxidative stress is however likely to be one factor in the pathogenesis of idiopathic $\mathrm{CH}$, suggesting antioxidants may have a role in the management of this disease.

Antioxidants include vitamin $\mathrm{C}$, vitamin $\mathrm{E}$, silymarin (silibinin or milk thistle extract) and $\mathrm{S}$ - 
adenosyl-L-methionine (SAMe). Vitamin $\mathrm{C}$ and $\mathrm{E}$ are normally synthesized by dogs, and it is not known whether deficiencies occur in canine idiopathic $\mathrm{CH}$ and therefore if supplementation is beneficial. Silymarin appears to be a strong free-radical scavenger, increasing normal cellular defense mechanisms against oxidative damage. In experimental laboratory animal studies it has been shown to protect against toxin induced liver damage, such as that due to acetaminophen. ${ }^{70}$ When given intravenously, its beneficial effects have also been reported in experimental amanita mushroom toxicity in dogs. ${ }^{71}$ S-adenosyl-Lmethionine is present in hepatocytes and is a precursor for cysteine, one of the amino acids of GSH. S-adenosyl-L-methionine is therefore important in the defense against oxygen free radicals. S-adenosyl-L-methionine is produced from methionine, which is activated by SAMe synthase in hepatocytes. In liver disease it is therefore possible that the production of SAMe and GSH may be reduced, although levels of GSH in canine liver disease vary. ${ }^{72}$ Administration of exogenous SAMe could therefore restore levels of GSH in hepatocytes. SAMe is also a critical enzyme in many biochemical reactions including the transmethylation, transsulfuration, and aminopropylation pathways, so is a key metabolite that regulates hepatocyte growth, death and differentiation. ${ }^{73}$ It is widely available as a nutraceutical for dogs, often combined with other antioxidants including silymarin. Sadenosyl-L-methionine in combination with silymarin has been shown to mitigate the apparent pro-oxidant influences of prednisolone ${ }^{74}$ and minimize the increase in liver enzymes in dogs receiving lomustine. ${ }^{75}$ There are no controlled studies reporting the effects of SAMe supplementation in canine idiopathic $\mathrm{CH}$, and there is limited evidence for the efficacy of SAMe in human chronic liver disease ${ }^{76}$ Having said that, due to the potential for oxidative stress and damage, and the fact that adverse effects at therapeutic dosages have not been reported, the author sometimes uses combinations of SAMe and silymarin in canine idiopathic $\mathrm{CH}$. 


\section{Diuretics}

Dogs with idiopathic $\mathrm{CH}$ and ascites formation may require diuretic therapy. Ascites is usually due to portal hypertension, although in some animals hypoalbuminemia may contribute to the pathogenesis. ${ }^{77}$ Portal hypertension results from an increased intrahepatic resistance combined with increased portal blood flow. Increased intrahepatic resistance results from fibrous tissue, sinusoidal endothelial dysfunction leading to impaired intrahepatic sinusoidal relaxation, and intrahepatic vascular shunts. Fluid is then driven into the interstitial space, and when the capacity of the regional lymphatics is overwhelmed, ascites develops. The development of ascites is worsened by the splanchnic vasodilation that accompanies portal hypertension. The subsequent reduction in systemic arterial blood pressure due to pooling of fluid in the abdomen activates the renin-angiotensin-aldosterone system (RAAS). Activation of the RAAS then leads to further fluid retention and more ascites. Spironolactone, an aldosterone antagonist, is therefore the drug of choice in ascitic dogs with idiopathic $\mathrm{CH}$ due to portal hypertension, usually at an initial dose of $2 \mathrm{mg} / \mathrm{kg} P O$ per day. Spironolactone can have a relatively slow onset of activity in humans, taking up to 14 days to causes diuresis; this may also occur in dogs with idiopathic $\mathrm{CH}$. In cases which are refractory to spironolactone, or when a more rapid resolution of ascites is required, furosemide (1-2 mg/kg $P O \mathrm{q} 12 \mathrm{~h})$ can also be used. However, therapy with furosemide has been shown to precipitate more complications in humans with ascites, especially electrolyte disturbances such as hypokalemia.

\section{Dietary management}

Appropriate dietary management is as important as drug therapy in the dog with idiopathic $\mathrm{CH}$. Each case is individual and the diet should be adjusted accordingly, so clinicians should 
resist the temptation to think that 'one diet fits all'. No studies have been performed in dogs with idiopathic $\mathrm{CH}$ to assess energy needs, or dietary composition, and recommendations are usually derived empirically from studies in humans, with most work having been done in patients with end-stage liver disease complicated by HE. ${ }^{78}$ It is the author's experience that many dogs with idiopathic $\mathrm{CH}$ are fed diets with inappropriate and excessive protein restriction, which may inhibit hepatic regeneration and result in protein-calorie malnutrition. Negative protein and energy balance in human patients with chronic liver disease has also been linked with abnormal immune responses, sepsis and mortality. ${ }^{79}$ If energy and protein metabolism of dogs with advanced liver disease are similar to those in humans, these patients actually have increased protein requirements, possibly two- or threefold maintenance values. ${ }^{80}$ Table 2 summarizes some of the important considerations in the dietary management of canine idiopathic $\mathrm{CH}$.

\section{Prognosis}

The prognosis for idiopathic $\mathrm{CH}$ is highly variable, and likely dependent on multiple factors, especially the stage of disease. The estimated median survival time of 43 dogs with idiopathic $\mathrm{CH}$ was 18.3 months, with a range of zero to 49 months. ${ }^{3}$ In Labrador retrievers with $\mathrm{CH}$, the median survival was 12.5 months (range, 1 day to 88 months), ${ }^{44}$ whereas in ESS it was 6.3 months (range, 1 day to 40.3 months). ${ }^{2}$ The presence of advanced fibrosis and cirrhosis are strongly associated with a reduced survival time in humans with $\mathrm{CH}$, and the presence of bridging fibrosis was a predictor of shorter survival time in dogs with $\mathrm{CH}$ of varying causes. ${ }^{57}$ Another important prognostic indictor in dogs with $\mathrm{CH}$ appears to be the presence of ascites; the time from onset of clinical signs to death was two months, compared to 33 months for dogs without ascites. ${ }^{81}$ A range of clinical pathology parameters have also been associated with a shorter survival time in dogs with $\mathrm{CH}$ including prolonged prothrombin 
time, prolonged partial thromboplastin time, thrombocytopenia, hypoalbuminemia, hyperbilirubinuria, and hyperglobulinemia. However caution should be employed when interpreting this data in the individual dog with idiopathic $\mathrm{CH}$, as prognosis is dependent on multiple variables. 


\section{Figure legends}

Figure 1: Photomicrograph of liver tissue from a Labrador retriever with idiopathic $\mathrm{CH}$ demonstrating several apoptotic hepatocytes within the hepatic parenchyma (arrows). Hematoxylin and eosin. Original magnification x 200 .

Figure 2: Photomicrograph of liver tissue from an English springer spaniel with idiopathic $\mathrm{CH}$ (same case as figure 1) demonstrating a foci of inflammatory cells in the hepatic parenchyma (arrow). Hematoxylin and eosin. Original magnification x 100.

Figure 3: Low-power photomicrograph of liver tissue from an English springer spaniel with idiopathic $\mathrm{CH}$ demonstrating disruption to the normal architecture by regenerative nodules (arrows). Hematoxylin and eosin. Original magnification x 40 .

Figure 4: Low-power photomicrograph of liver tissue from a Doberman pinscher with idiopathic $\mathrm{CH}$ demonstrating marked fibrosis (light green staining). Masson's trichrome. Original magnification x 40 . 
Table 1. Age and gender data for breeds with an increased risk for developing canine $\mathrm{CH}$.

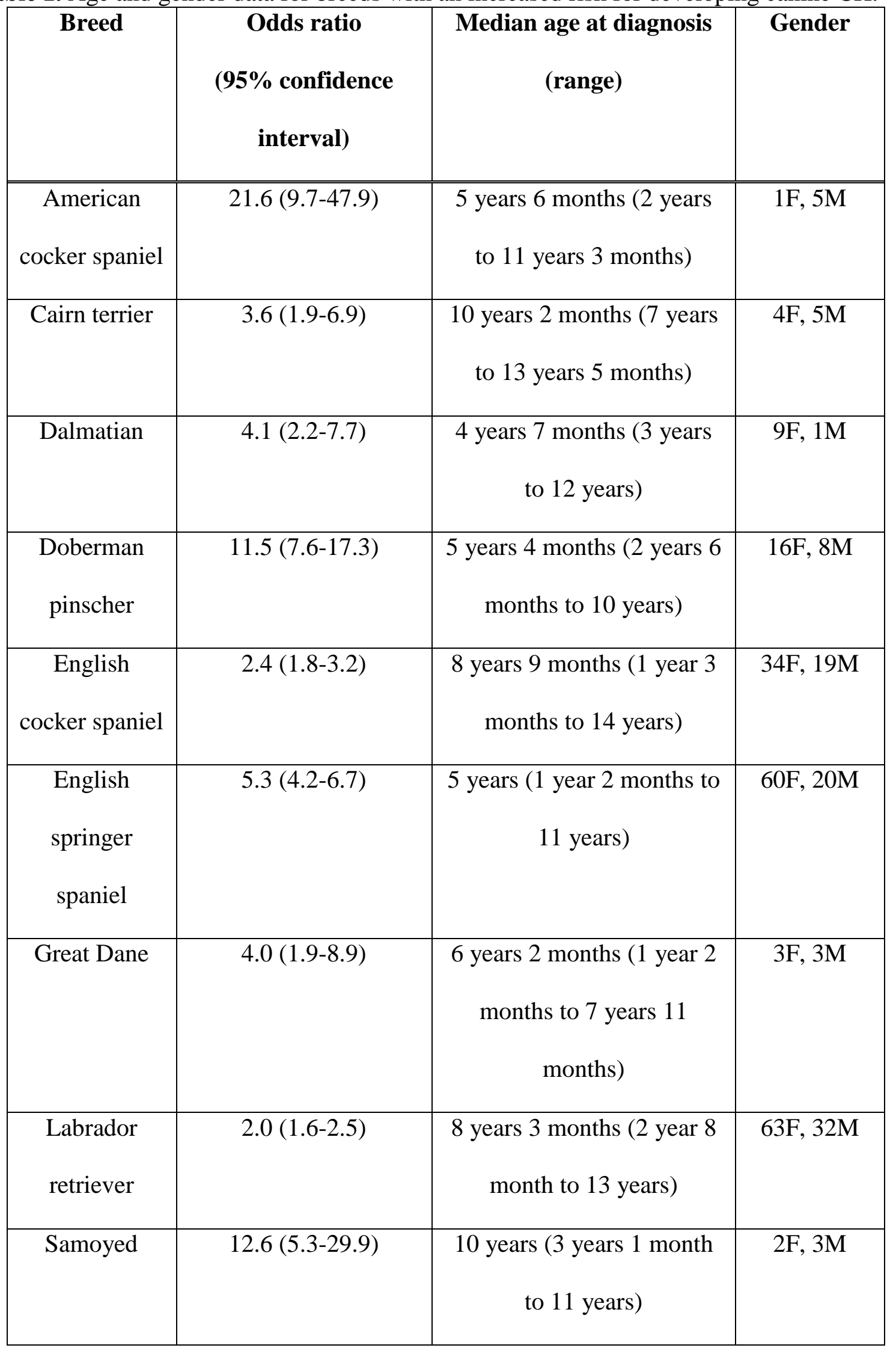

M, male; F, female. Odds ratios are given based on 2008 control data. 
Table 2. Summary of the dietary recommendations for canine idiopathic $\mathrm{CH}$.

\begin{tabular}{|c|c|}
\hline $\begin{array}{l}\text { Palatability and } \\
\text { frequency }\end{array}$ & $\begin{array}{l}\text { As dogs with idiopathic } \mathrm{CH} \text { may be inappetent, feed a palatable diet } \\
\text { several times per day. Frequent feeding maximizes energy intake and } \\
\text { helps to reduce the development of hepatic encephalopathy }\end{array}$ \\
\hline Protein & $\begin{array}{l}\text { As detailed above protein restriction should be avoided in dogs with } \\
\text { idiopathic } \mathrm{CH} \text {, and only done if necessary to control signs of hepatic } \\
\text { encephalopathy. Excessive protein restriction may result in } \\
\text { protein:calorie malnutrition and the breakdown of highly } \\
\text { ammoniaogenic endogenous proteins. Feed highly digestible, high } \\
\text { quality protein such as that from vegetable or casein sources. Regularly } \\
\text { assess weight, muscle mass and blood albumin }\end{array}$ \\
\hline Carbohydrates & $\begin{array}{l}\text { Dogs with idiopathic } \mathrm{CH} \text { may have impaired carbohydrate metabolism, } \\
\text { so a diet containing highly digestible, complex carbohydrates should be } \\
\text { fed. }\end{array}$ \\
\hline Fat & $\begin{array}{l}\text { Normal amounts of fat should be fed, although fat can be restricted if } \\
\text { steatorrhea develops }\end{array}$ \\
\hline Fiber & $\begin{array}{l}\text { Fermentable fiber is helpful in dogs with hepatic encephalopathy as it } \\
\text { acidifies the colon and traps ammonia. It also increases nitrogen } \\
\text { incorporation into bacteria and reduces bacterial ammonia production. } \\
\text { Non-fermentable fibre is helpful in preventing constipation, a } \\
\text { predisposing factor for hepatic encephalopathy. }\end{array}$ \\
\hline Zinc & $\begin{array}{l}\text { Zinc is essential to large numbers of metalloenzymes involved in a } \\
\text { range of biochemical processes. Zinc is also involved in membrane } \\
\text { stability and has free radical and anti-oxidant effects. Zinc metabolism } \\
\text { may become disrupted in patients with liver disease. Zinc deficiency } \\
\text { occurs in human } \mathrm{CH} \text { and may occur in dogs with idiopathic } \mathrm{CH} \text {, and so } \\
\text { supplementation may be required. }\end{array}$ \\
\hline $\begin{array}{l}\text { Fat-soluble } \\
\text { vitamins }\end{array}$ & $\begin{array}{l}\text { These include A, D, E, and K. Malabsorption of fat-soluble vitamins } \\
\text { can occur with disruption to the enterohepatic circulation of bile acids. } \\
\text { As detailed above, Vitamin E is an antioxidant and can be } \\
\text { supplemented in dogs with idiopathic CH. Vitamin K supplementation } \\
\text { may be necessary if coagulation times are prolonged, especially }\end{array}$ \\
\hline
\end{tabular}




\begin{tabular}{|l|l|}
\hline & $\begin{array}{l}\text { proceeding biopsy. Vitamins A and D should not probably be } \\
\text { supplemented as excess Vitamin A can cause hepatic damage and } \\
\text { excess vitamin D may result in hypercalcaemia. }\end{array}$ \\
\hline $\begin{array}{l}\text { Water soluble } \\
\text { vitamins }\end{array}$ & $\begin{array}{l}\text { Thiamine (Vitamin B-1) is an essential coenzyme in carbohydrate } \\
\text { metabolism and deficiency can results in hepatic encephalopathy-like } \\
\text { signs. Supplementation with Vitamin C is not recommended. }\end{array}$ \\
\hline
\end{tabular}




\section{References}

1. Van den Ingh TSGAM, Van Winkle TJ, Cullen JM, et al. Morphological classification of parenchymal disorders of the canine and feline liver: 2 Hepatocellular death, hepatitis and cirrhosis In: Rothuizen J, Bunch SE, Charles JA, et al., editors. WSAVA Standards for clinical and histological diagnosis of canine and feline liver disease. 1st edition. Philadelphia, PA: Saunders Elsevier, 2006;p. 85-102.

2. Bexfield NH, Andres-Abdo C, Scase TJ, et al. Chronic hepatitis in the English springer spaniel: clinical presentation, histological description and outcome. Vet Rec 2011;169:415.

3. Poldervaart JH, Favier RP, Penning LC, et al. Primary hepatitis in dogs: a retrospective review (2002-2006). J Vet Intern Med 2009;23:72-80.

4. Knodell RG, Ishak KG, Black WC, et al. Formulation and application of a numerical scoring system for assessing histological activity in asymptomatic chronic active hepatitis. Hepatology 1981;1:431-435.

5. Ishak K, Baptista A, Bianchi L, et al. Histological grading and staging of chronic hepatitis. J Hepatol 1995;22:696-699.

6. Boisclair J, Dore M, Beauchamp G, et al. Characterization of the inflammatory infiltrate in canine chronic hepatitis. Vet Pathol 2001;38:628-635.

7. Sakai M, Otani I, Ishigaki K, et al. Phenotypic analysis of hepatic T lymphocytes in a dog with chronic hepatitis. J Vet Med Sci 2006;68:1219-1221.

8. Friedman SL. Seminars in medicine of the Beth Israel Hospital, Boston. The cellular basis of hepatic fibrosis. Mechanisms and treatment strategies. $N$ Engl J Med 1993;328:1828-1835.

9. Reynaert H, Thompson MG, Thomas T, et al. Hepatic stellate cells: role in microcirculation and pathophysiology of portal hypertension. Gut 2002;50:571-581.

10. Benyon RC, Iredale JP. Is liver fibrosis reversible? Gut 2000;46:443-446.

11. Bataller R, Brenner DA. Hepatic stellate cells as a target for the treatment of liver fibrosis. Semin Liver Dis 2001;21:437-451.

12. Ijzer J, Roskams T, Molenbeek RF, et al. Morphological characterisation of portal myofibroblasts and hepatic stellate cells in the normal dog liver. Comp Hepatol 2006;5:7.

13. Ljubuncic P, Tanne Z, Bomzon A. Evidence of a systemic phenomenon for oxidative stress in cholestatic liver disease. Gut 2000;47:710-716.

14. Watson PJ. Chronic hepatitis in dogs: a review of current understanding of the aetiology, progression, and treatment. Vet $J$ 2004;167:228-241.

15. Bexfield NH, Buxton RJ, Vicek TJ, et al. Breed, age and gender distribution of dogs with chronic hepatitis in the United Kingdom. Vet J 2012.

16. Gocke DJ, Morris TQ, Bradley SE. Chronic hepatitis in the dog: the role of immune factors. J Am Vet Med Assoc 1970;156:1700-1705.

17. Bishop L, Strandberg JD, Adams RJ, et al. Chronic active hepatitis in dogs associated with leptospires. Am J Vet Res 1979;40:839-844.

18. Adamus C, Buggin-Daubie M, Izembart A, et al. Chronic hepatitis associated with leptospiral infection in vaccinated beagles. J Comp Pathol 1997;117:311-328.

19. Kapoor A, Simmonds P, Gerold G, et al. Characterization of a canine homolog of hepatitis C virus. Proceedings of the National Academy of Sciences of the United States of America 2011;108:11608-11613.

20. Bexfield NH, Watson PJ, Heaney J, et al. Canine hepacivirus is not associated with chronic liver disease in dogs. Journal of viral hepatitis 2014;21:223-228.

21. van der Laan LJ, de Ruiter PE, van Gils IM, et al. Canine hepacivirus and 
idiopathic hepatitis in dogs from a Dutch cohort. Journal of viral hepatitis 2014;21:894-896.

22. O'Neill EJ, Day MJ, Hall EJ, et al. Bacterial cholangitis/cholangiohepatitis

with or without concurrent cholecystitis in four dogs. J Small Anim Pract 2006;47:325-335.

23. Tamborini A, Jahns H, McAllister H, et al. Bacterial Cholangitis, Cholecystitis, or both in Dogs. J Vet Intern Med 2016.

24. Twedt DC, Diehl KJ, Lappin MR, et al. Association of hepatic necrosis with trimethoprim sulfonamide administration in 4 dogs. $J$ Vet Intern Med 1997;11:20-23.

25. Bunch SE, Castleman WL, Hornbuckle WE, et al. Hepatic cirrhosis associated with long-term anticonvulsant drug therapy in dogs. J Am Vet Med Assoc 1982;181:357-362.

26. Hardy RM, Stevens JB, Stowe CM. Chronic progressive hepatitis in Bedlington terriers associated with elevated liver copper concentrations. Minnesota Veterinarian 1975;15:13-24.

27. Webb CB, Twedt DC, Meyer DJ. Copper-associated liver disease in Dalmatians: a review of 10 dogs (1998-2001). J Vet Intern Med 2002;16:665-668.

28. Mandigers PJ, van den Ingh TS, Bode P, et al. Association between liver copper concentration and subclinical hepatitis in Doberman Pinschers. $J$ Vet Intern Med 2004; 18:647-650.

29. Hoffmann $\mathrm{G}$, van den Ingh TS, Bode $\mathrm{P}$, et al. Copper-associated chronic hepatitis in Labrador Retrievers. J Vet Intern Med 2006;20:856-861.

30. Andersson M, Sevelius E. Circulating autoantibodies in dogs with chronic liver disease. J Small Anim Pract 1992;33:389-394.

31. Weiss DJ, Armstrong PJ, Mruthyunjaya A. Anti-liver membrane protein antibodies in dogs with chronic hepatitis. J Vet Intern Med 1995;9:267-271.

32. Dyggve H, Jarva H, Spillmann T, et al. Autoantibodies in Dobermann Hepatitis. ECVIM-CA Congress. Toulouse, France, 2010.

33. Dyggve H, Meri S, Spillmann T, et al. Anti-Nuclear Antibodies in Doberman Hepatitis. ECVIM-CA Congress. Seville, Spain, 2011.

34. Dyggve H, Meri S, Spillmann T, et al. Anti-Histone Antibodies in Doberman Hepatitis. ECVIM-CA Congress. Maastricht, Netherlands, 2012.

35. Dyggve H, Kennedy LJ, Meri S, et al. Association of Doberman hepatitis to canine major histocompatibility complex II. Tissue antigens 2011;77:30-35.

36. Bexfield NH, Watson PJ, Aguirre-Hernandez J, et al. DLA class II alleles and haplotypes are associated with risk for and protection from chronic hepatitis in the English Springer spaniel. PloS one 2012;7:e42584.

37. Watson PJ, Roulois AJ, Scase TJ, et al. Prevalence of hepatic lesions at postmortem examination in dogs and association with pancreatitis. J Small Anim Prac 2010;51:566-572.

38. Andersson M, Sevelius E. Breed, sex and age distribution in dogs with chronic liver disease: a demographic study. J Small Anim Pract 1991;32:1-5.

39. Hirose N, Uchida K, Kanemoto H, et al. A retrospective histopathological survey on canine and feline liver diseases at the University of Tokyo between 2006 and 2012. J Vet Med Sci 2014;76:1015-1020.

40. Center SA. Pathophysiology of Liver Disease: Normal and Abnormal function In: Guilford WG, Center SA, Strombeck DR, et al., editors. Strombeck's Small Animal Gastroenterology. 3rd edition. Philadelphia: W.B. Saunders Company, 1996;553-632.

41. Szawlowski AW, Saint-Aubert B, Gouttebel MC, et al. Experimental model of extended repeated partial hepatectomy in the dog. Eur Surg Res 1987;19:375-380.

42. Watson PJ, Bunch SE. Hepatobiliary Diseases in the Dog In: Nelson RW, Couto CG, editors. Small Animal Internal Medicine. 4th edition. St. Louis: Mosby Elsevier, 2009. 
43. Webster CRL. History, Clinical Signs, and Physical Findings in Hepatobiliary Disease In: Ettinger SJ,Feldman EC, eds. Textbook of Veterinary Internal Medicine. 7th ed. St. Louis: Saunders Elsevier, 2010;1612-1626.

44. Shih JL, Keating JH, Freeman LM, et al. Chronic hepatitis in Labrador Retrievers: clinical presentation and prognostic factors. $J$ Vet Intern Med 2007;21:33-39.

45. Prater MR. Acquired coagulopathy II: liver disease In: Feldman BF, Zinkl JG,N.C. J, eds. Schalm's Veterinary Hematology. Philadelphia, PA: Williams and Wilkins, 2000;560-564.

46. Mammen EF. Coagulation abnormalities in liver disease. Hematology/oncology clinics of North America 1992;6:1247-1257.

47. Prins M, Schellens CJ, van Leeuwen MW, et al. Coagulation disorders in dogs with hepatic disease. Vet $J$ 2010;185:163-168.

48. Schwarz T. The liver and gallbladder In: O'Brien RT,Barr F, editors. BSAVA Manual of Canine and Feline Abdominal Imaging. Gloucester: BSAVA Publications, 2009;p. 144-156.

49. Warren-Smith CM, Andrew S, Mantis P, et al. Lack of associations between ultrasonographic appearance of parenchymal lesions of the canine liver and histological diagnosis. J Small Anim Pract 2012;53:168-173.

50. Feeney DA, Anderson KL, Ziegler LE, et al. Statistical relevance of ultrasonographic criteria in the assessment of diffuse liver disease in dogs and cats. Am J Vet Res 2008;69:212-221.

51. D'Anjou M. Liver In: D'Anjou M,Pennick D, eds. Atlas of Small Animal Ultrasonography. 1st ed: Blackwell Publishing, 2008;217-262.

52. Cole TL, Center SA, Flood SN, et al. Diagnostic comparison of needle and wedge biopsy specimens of the liver in dogs and cats. J Am Vet Med Assoc 2002;220:14831490.

53. Wang KY, Panciera DL, Al-Rukibat RK, et al. Accuracy of ultrasound-guided fine-needle aspiration of the liver and cytologic findings in dogs and cats: 97 cases (19902000). J Am Vet Med Assoc 2004;224:75-78.

54. Bigge LA, Brown DJ, Penninck DG. Correlation between coagulation profile findings and bleeding complications after ultrasound-guided biopsies: 434 cases (1993-1996). J Am Anim Hosp Assoc 2001;37:228-233.

55. Recordati C, Gualdi V, Craven M, et al. Spatial distribution of Helicobacter spp. in the gastrointestinal tract of dogs. Helicobacter 2009;14:180-191.

56. Rhen T, Cidlowski JA. Antiinflammatory action of glucocorticoids--new mechanisms for old drugs. The New England journal of medicine 2005;353:1711-1723.

57. Strombeck DR, Miller LM, Harrold D. Effects of corticosteroid treatment on survival time in dogs with chronic hepatitis: 151 cases (1977-1985). J Am Vet Med Assoc 1988;193:1109-1113.

58. Cocco G, Chu DC, Pandolfi S. Colchicine in clinical medicine. A guide for internists. European journal of internal medicine 2010;21:503-508.

59. Lange U, Schumann C, Schmidt KL. Current aspects of colchicine therapy -classical indications and new therapeutic uses. European journal of medical research 2001;6:150-160.

60. Friedman SL. Evolving challenges in hepatic fibrosis. Nature reviews Gastroenterology \& hepatology 2010;7:425-436.

61. Rutgers HC, Haywood S, Kelly DF. Idiopathic hepatic fibrosis in 15 dogs. Vet Rec 1993;133:115-118.

62. Hill PB, Auxilia ST, Munro E, et al. Resolution of skin lesions and long-term survival in a dog with superficial necrolytic dermatitis and liver cirrhosis. J Small Anim Pract 
2000;41:519-523.

63. Guldutuna S, Zimmer G, Imhof M, et al. Molecular aspects of membrane stabilization by ursodeoxycholate [see comment]. Gastroenterology 1993;104:1736-1744.

64. Rodrigues CM, Fan G, Ma X, et al. A novel role for ursodeoxycholic acid in inhibiting apoptosis by modulating mitochondrial membrane perturbation. The Journal of clinical investigation 1998;101:2790-2799.

65. Yanaura S, Ishikawa S. Choleretic properties of ursodeoxycholic acid and chenodeoxycholic acid in dogs. Japanese journal of pharmacology 1978;28:383-389.

66. Meyer DJ, Thompson MB, Senior DF. Use of ursodeoxycholic acids in a dog with chronic hepatitis: effects on serum hepatic tests and endogenous bile acid composition. $J$ Vet Intern Med 1997;11:195-197.

67. Noda T, Mimura H, Orita K. Assessment of Kupffer cell function in rats with chronic liver injury caused by CCl4. Hepato-gastroenterology 1990;37:319-323.

68. Bexfield N. Ascites and Hepatic Encephalopathy Therapy for Liver Disease In: Bonagura JD,Twedt DC, editors. Kirk's Current Veterinary Therapy XV. St. Louis, Missouri: Elsevier Saunders, 2014;p. 591-594.

69. Song Z, McClain CJ, Chen T. S-Adenosylmethionine protects against acetaminophen-induced hepatotoxicity in mice. Pharmacology 2004;71:199-208.

70. Muriel P, Garciapina T, Perez-Alvarez V, et al. Silymarin protects against paracetamol-induced lipid peroxidation and liver damage. Journal of applied toxicology : JAT 1992;12:439-442.

71. Vogel G, Tuchweber B, Trost W, et al. Protection by silibinin against Amanita phalloides intoxication in beagles. Toxicology and applied pharmacology 1984;73:355-362.

72. Center SA, Warner KL, Erb HN. Liver glutathione concentrations in dogs and cats with naturally occurring liver disease. Am J Vet Res 2002;63:1187-1197.

73. Mato JM, Lu SC. Role of S-adenosyl-L-methionine in liver health and injury. Hepatology 2007;45:1306-1312.

74. Center SA, Warner KL, McCabe J, et al. Evaluation of the influence of Sadenosylmethionine on systemic and hepatic effects of prednisolone in dogs. Am J Vet Res 2005;66:330-341.

75. Skorupski KA, Hammond GM, Irish AM, et al. Prospective randomized clinical trial assessing the efficacy of Denamarin for prevention of CCNU-induced hepatopathy in tumor-bearing dogs. J Vet Intern Med 2011;25:838-845.

76. Guo T, Chang L, Xiao Y, et al. S-adenosyl-L-methionine for the treatment of chronic liver disease: a systematic review and meta-analysis. PloS one 2015;10:e0122124.

77. Buob S, Johnston AN, Webster CR. Portal hypertension: pathophysiology, diagnosis, and treatment. J Vet Intern Med 2011;25:169-186.

78. Center SA. Nutritional support for dogs and cats with hepatobiliary disease. The Journal of nutrition 1998;128:2733S-2746S.

79. O'Keefe SJ, El-Zayadi AR, Carraher TE, et al. Malnutrition and immunoincompetence in patients with liver disease. Lancet 1980;2:615-617.

80. Elwyn DH. Protein metabolism and requirements in the critically ill patient. Critical care clinics 1987;3:57-69.

81. Raffan E, McCallum A, Scase TJ, et al. Ascites is a negative prognostic indicator in chronic hepatitis in dogs. J Vet Intern Med 2009;23:63-66. 